\title{
Subband Merging in Cosine-Modulated Filter Banks
}

\author{
Omar A. Niamut and Richard Heusdens
}

\begin{abstract}
Recently, a new method for constructing nonuniform modulated lapped transforms (MLTs) was introduced, by combining subband filters of a uniform MLT. The design, however, was restricted to combining two or four subband filters only, and no systematic design procedure was given. In this letter, we propose an extension to the above-mentioned method that allows arbitrary numbers of subbands to be combined in a systematic way. We investigate the general case of combining filters in arbitrary cosine-modulated filter banks, and give conditions on how to combine the constituent filters such that the resulting nonuniform filter banks have suitable frequency responses.
\end{abstract}

Index Terms-Lapped transforms, modulated filter banks, nonuniform filter banks.

\section{INTRODUCTION}

$\mathbf{R}$ ECENTLY, a new method for constructing nonuniform modulated lapped transforms (MLTs) was introduced [1], [2] by combining subband filters of a uniform MLT. We shall refer to this method as subband merging. The combined subband filters have better time localization than the noncombined filters at the expense of a decrease in frequency localization. Since the nonuniform filter banks are obtained by simply taking linear combinations of the filters of a uniform MLT, the proposed method allows for an efficient implementation of time-varying transforms without the need for transition filters. It was shown that subband merging can be used beneficially for reducing ringing artifacts, e.g., reverberation and pre-echo, in audio and speech coding [2]. The design of such transforms, however, was restricted to combining two or four subband filters only, and no systematic design procedure was given.

Alternative methods similar to subband merging do exist. In [3] and [4], a method for designing nonuniform filter banks is presented by postprocessing a number of selected subbands using a special synthesis bank. However, the method described in [3] leads, in general, to more complex solutions than the subband merging approach, while the method described in [4] leads to signal-dependent subband filters. In [5] and [6], a number of subbands are added together, and the decimation factor is changed accordingly, resulting in nonperfect reconstruction filter banks, while [7] describes a method of combining filters of a modulated complex lapped transform. The latter, however, is less suited for coding applications due to the inherent oversampling.

Manuscript received March 7, 2002; revised August 29, 2002. This work was supported by Philips Research and the Technology Foundation STW, applied science division of NWO and the Ministry of Economics Affairs. The associate editor coordinating the review of this manuscript and approving it for publication was Prof. Dimitris A. Pados.

The authors are with the Department of Mediamatics, Delft University of Technology, 2628 CD Delft, The Netherlands (e-mail: O.A.Niamut@ its.tudelft.nl).

Digital Object Identifier 10.1109/LSP.2003.809032
In this letter, we propose an extension of the above-mentioned subband merging method [1], [2] that allows an arbitrary number of subbands to be combined in a systematic way. We investigate the more general case of combining filters in arbitrary cosine-modulated filter (CMF) banks. Starting from a uniform CMF bank, we take linear combinations of the constituent filters such that the resulting combined filters have good frequency-selective properties and flat passband response.

\section{UNIFORM CMF BANKS}

In an $M$-channel maximally decimated CMF bank, the filters are derived by cosine modulation of a single prototype filter $p_{0}$. Let $p_{0}$ be a real-coefficient linear-phase lowpass filter of length $N$. A closed-form expression of the impulse response $h_{k}(n)$ of the analysis filters is then given by [8]

$$
\begin{aligned}
h_{k}(n)=2 p_{0}(n) \cos \left[\frac{\pi}{M}\left(k+\frac{1}{2}\right)\left(n-\frac{\alpha}{2}\right)\right], \\
n=0, \ldots, N-1
\end{aligned}
$$

where $\alpha \in \mathbb{Z}$ is called the modulation phase. By choosing the synthesis filters $F_{k}(z)$ according to the mirror image condition [9]

$$
F_{k}(z)=z^{-n_{0}} \tilde{H}_{k}(z)
$$

for some $n_{0} \in \mathbb{Z}$ ( ${ }^{\sim}$ denotes paraconjugation), we can write the CMF bank transfer functions $T_{\ell}(z)$ as

$T_{\ell}(z)=\frac{z^{-n_{0}}}{M} \sum_{k=0}^{M-1} H_{k}\left(z W^{\ell}\right) \tilde{H}_{k}(z), \quad \ell=0, \ldots, M-1$

where $W=e^{-j 2 \pi / M}$. For perfect reconstruction (PR), we have that the aliasing transfer functions $T_{\ell}(z)=0$ for $1 \leq \ell \leq M$ and that the distortion transfer function

$$
T_{0}(z)=\frac{z^{-n_{0}}}{M} \sum_{k=0}^{M-1} H_{k}(z) \tilde{H}_{k}(z) \equiv c z^{-n_{0}}, \quad c \neq 0
$$

i.e., the output of the filter bank is a scaled and delayed version of the input. The PR property can be satisfied by suitable choices of $\alpha$ and the prototype filter $p_{0}$.

\section{Design OF NONUNIFORM CMF BANKS}

We investigate the case where an arbitrary number, say $p \leq$ $M$, of adjacent filters in a uniform CMF bank are combined. For the nonuniform filter bank to have a suitable frequency response, the magnitude characteristics of its filters must exhibit good frequency selectivity and flat passband response. We, therefore, derive necessary and sufficient conditions on the underlying uniform CMF bank and the way to combine the con- 


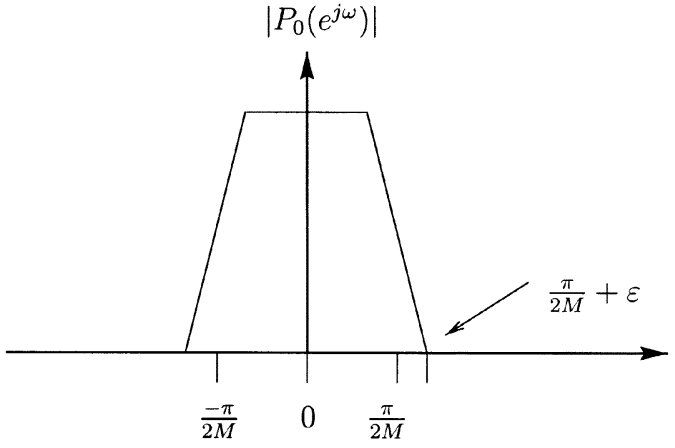

Fig. 1. Magnitude response of the prototype filter $p_{0}$.

stituent filters such that the above-mentioned requirements are satisfied.

Let $p_{0}$ be a real-coefficient linear-phase lowpass filter of length $N$ with passband $[-(\pi / 2 M)+\varepsilon,(\pi / 2 M)-\varepsilon), \varepsilon<$ $\pi / 2 M$, and an infinitely attenuated stopband (see Fig. 1), i.e.,

$$
\left|P_{0}\left(e^{j \omega}\right)\right|=0 \text { for }|\omega| \geq \frac{\pi}{2 M}+\varepsilon, \varepsilon<\frac{\pi}{2 M}
$$

and let $H_{p, k}(z)$ be a linear combination of $p$ adjacent filters starting from the $k$ th filter in a uniform CMF bank, i.e.,

$$
H_{p, k}(z)=\sum_{i=0}^{p-1} b_{k+i} H_{k+i}(z), \quad k=0, \ldots, M-p
$$

with $b_{k}=e^{j \varphi_{k}}$ the combinatorial coefficients of magnitude one. If $\left|H_{p, k}(z)\right|^{2}$ (or equivalently $\left|\sum_{i=0}^{p-1} b_{k+i} H_{k+i}(z)\right|^{2}$ ) is equal to $\sum_{i=0}^{p-1}\left|H_{k+i}(z)\right|^{2}$, then $H_{p, k}(z)$ has flat passband response and a transition bandwidth similar to those of the underlying uniformly spaced subband filters. This can be easily seen by inspection of (2) and the fact that there is no spectral overlap between filters $H_{k}(z)$ and $H_{l}(z)$ for $|k-l| \geq 2$, so that $\sum_{i=0}^{p-1}\left|H_{k+i}\left(e^{j \omega}\right)\right|^{2}=c, \quad c \neq 0$, for $|\omega| \in[(k \pi / M)+$ $\varepsilon,((k+p) \pi / M)-\varepsilon)$, and zero in its stopband. The following theorem gives necessary and sufficient conditions on the modulation phase (i.e., on the uniform filter bank) and the combinatorial coefficients such that the resulting combined filters indeed exhibit the required frequency behavior.

Theorem 1: Let $p_{0}$ denote a real-coefficient linear-phase lowpass prototype filter of length $N$ for an $M$-channel PR uniform CMF bank, satisfying

$$
\left|P_{0}\left(e^{j \omega}\right)\right|=0 \text { for }|\omega| \geq \frac{\pi}{2 M}+\varepsilon, \varepsilon<\frac{\pi}{2 M}
$$

and let $b_{k}=e^{j \varphi_{k}}, k=0, \ldots, M-1$.

We then have

$$
\left|\sum_{i=0}^{p-1} b_{k+i} H_{k+i}(z)\right|^{2}=\sum_{i=0}^{p-1}\left|H_{k+i}(z)\right|^{2}
$$

for $1 \leq p \leq M$ and $0 \leq k \leq M-p$, if and only if $\alpha=$ $(N-1)-M(2 m+1), m \in \mathbb{Z}$, and $\left|\varphi_{k}-\varphi_{k+1}\right|=n \pi, n \in \mathbb{N}$.

Proof: See [10].

Some remarks are in place here. The condition (3) on the prototype filter $p_{0}$ can never be satisfied in practical appli- cations, since it requires infinite length filters. Therefore, in practical situations, overlapping terms in the frequency domain of nonadjacent filters do exist and will result in ripples in the passband of the combined filters. However, by keeping the stopband attenuation of the prototype filter high, these ripples are kept to a minimum. Moreover, the condition on $\alpha$ might seem a new restriction on the underlying uniform CMF bank, but this is not the case. Most CMF banks known from literature (e.g., the MLT, as well as the ones reported in [9]) satisfy the condition on $\alpha$, since it cancels first-order aliasing and magnitude distortion at $\omega \in\{0, \pi\}$. As for the condition on $b_{k}$, this amounts to choosing combinatorial coefficients that can only differ in sign.

The combination operation can be represented by a matrix multiplication. Consider the example in which we combine two filters $(p=2)$ according to the method in [1]. If we define the matrix $\mathbf{A} \in \mathbb{C}^{M \times N}$ containing the impulse responses of the analysis filters of the uniform CMF bank as

$$
\mathbf{A}=\left(\begin{array}{ccc}
h_{0}(0) & \ldots & h_{0}(N-1) \\
\vdots & \ddots & \vdots \\
h_{M-1}(0) & \ldots & h_{M-1}(N-1)
\end{array}\right)
$$

we can create the matrix $\mathbf{A}^{\prime}$, which contains the impulse responses of the analysis filters of the nonuniform CMF bank, by the matrix multiplication $\mathbf{A}^{\prime}=\mathbf{S A}$, where

$$
\mathbf{S}=\left(\begin{array}{rrrrrr}
1 & & & & & \\
& \ddots & & & \varnothing & \\
& & 1 & 1 & & \\
& & 1 & -1 & & \\
& \varnothing & & & \ddots & \\
& & & & & 1
\end{array}\right) \in \mathbb{C}^{M \times M} .
$$

The combinatorial coefficients $b_{k}$ are found in the rows of the block-diagonal element of $\mathbf{S}$, which in this case is a size two Hadamard matrix. Hence, $\mathbf{S}$ is clearly nonsingular.

In the remainder of this section, we consider the case $p \geq 2$. In this case, the nonsingular block-diagonal element in $\mathbf{S}$ is of size $p \times p$ having entries $b_{k}$. Although many matrices exist that satisfy the constraints on the $b_{k}$ 's, we propose a simple construction method that results in real-coefficient integer matrices. We can obtain a suitable nonsingular matrix by taking the $p \times p$ principal submatrix of a size $N \geq p$ Hadamard matrix. To show that such a submatrix is indeed nonsingular, we need the following theorem (proven in the Appendix).

Theorem 2: Let $\mathbf{X} \in \mathbb{C}^{N \times N}$ such that every principal submatrix of $\mathbf{X}$ is nonsingular. Let $\mathbf{Y} \in \mathbb{C}^{2 N \times 2 N}$ be constructed as

$$
\mathbf{Y}=\left(\begin{array}{rr}
\mathbf{X} & \mathbf{X} \\
\mathbf{X} & -\mathbf{X}
\end{array}\right)
$$

Then every principal submatrix of $\mathbf{Y}$ is nonsingular.

Applying Theorem 2 to Hadamard matrices we obtain the desired result.

Corollary 1: Let $\mathbf{H}_{N} \in \mathbb{C}^{N \times N}$ be a Hadamard matrix of size $N=2^{n}, n \geq 1$. Then every principal submatrix of $\mathbf{H}_{N}$ is nonsingular. 

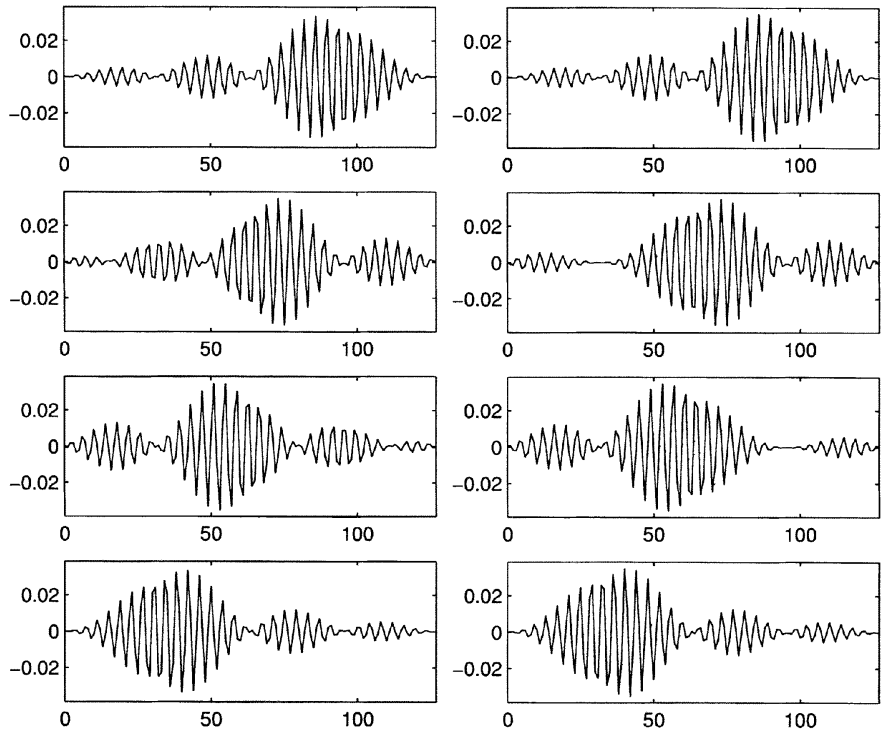

Fig. 2. Time-domain responses of four combined subband filters of a 64-channel MLT for (a) the method reported in [2] and (b) the newly proposed method.

Proof: Since $\mathbf{H}_{N}$ is Hadamard, it is constructed as

$$
\mathbf{H}_{N}=\left(\begin{array}{cc}
\mathbf{H}_{N / 2} & \mathbf{H}_{N / 2} \\
\mathbf{H}_{N / 2} & -\mathbf{H}_{N / 2}
\end{array}\right)
$$

so that every principal submatrix of $\mathbf{H}_{N}$ is nonsingular by Theorem 2.

The design of a PR nonuniform CMF banks comes down to representing the desired filter bank structure as nonsingular blocks in the matrix $\mathbf{S}$, chosen according to the method described above. The matrix thus obtained is invertible and, for $p=2^{n}, \quad n \in \mathbb{N}$, can be made unitary by scaling the $b_{k}$ 's properly, so that, assuming the original uniform filter bank is unitary, the nonuniform filter bank is unitary as well. In this sense, subband merging can be seen as a special case of unitary postprocessing [3], [4]. However, our approach gives signal-independent integer postprocessing matrices that allow for efficient implementation with low computational complexity.

\section{EXPERIMENTAL RESULTS}

In order to compare our results to the ones reported in [2], we combined four subband filters in a 64-channel MLT. The resulting time and frequency responses of the combined filters are shown in Figs. 2 and 3, respectively. Fig. 2(a) and 3(a) show results reported in [2], while Fig. 2(b) and 3(b) show results for the newly proposed method. By inspection of the figures, it can be seen that for comparable time localization, the new method gives better frequency responses.
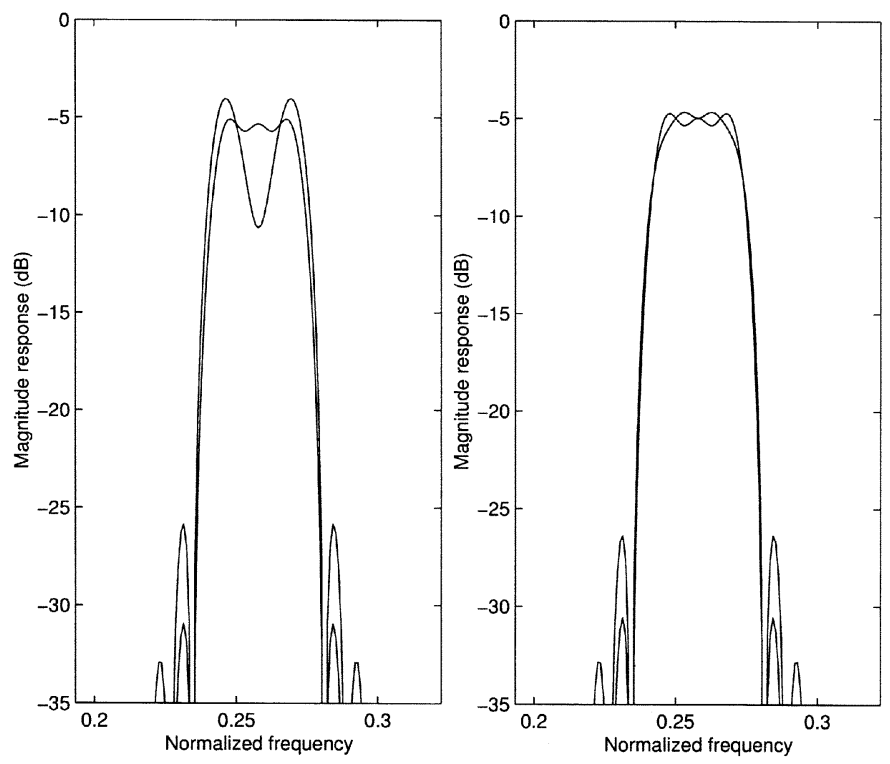

Fig. 3. Magnitude responses of four combined subband filters of a 64-channel MLT for (a) the method reported in [2] and (b) the newly proposed method.

\section{APPENDIX \\ PROOF OF THEOREM 2}

Proof: The proof goes by induction to $p$, the size of the principal submatrix. For $1 \leq p \leq N$, the hypothesis is true by definition. Let $p=N+a, 1 \leq a \leq N$. We then have a principal submatrix $\mathbf{P}$ of size $p=N+a$ of the following structure:

$$
\mathbf{P}=\left(\begin{array}{rrr}
\mathbf{A} & \mathbf{B} & \mathbf{A} \\
\mathbf{C} & \mathbf{D} & -\mathbf{C}
\end{array}\right)
$$

where $\mathbf{A} \in \mathbb{C}^{N \times a}, \mathbf{B} \in \mathbb{C}^{N \times(N-a)}, \mathbf{C} \in \mathbb{C}^{a \times a}$, and $\mathbf{D} \in$ $\mathbb{C}^{a \times(N-a)}$. Let $\mathbf{P z}=\mathbf{o}$, the zero element, for some $\mathbf{z} \in \mathbb{C}^{p}$. Since the first $N$ elements of $\mathbf{P z}$ are linear combinations of the columns of $\mathbf{A}$ and $\mathbf{B}$ only, and the fact that the intersection of the subspaces spanned by these columns is $\mathbf{o}$, we conclude that $\mathbf{z}$ is of the form

$$
\mathbf{z}=\left(\begin{array}{r}
\mathbf{z}_{a} \\
\mathbf{o} \\
-\mathbf{z}_{a}
\end{array}\right)
$$

where $\mathbf{z}_{a} \in \mathbb{C}^{a}$ and $\mathbf{o} \in \mathbb{C}^{N-a}$. From this, the last $a$ elements of $\mathbf{P z}$ can be expressed as $\mathbf{o}=2 \mathbf{C z}_{a}$, from which we conclude that $\mathbf{z}_{a}=\mathbf{o}$, since $\mathbf{C}$ is nonsingular by the induction hypothesis.

\section{REFERENCES}

[1] H. S. Malvar, "Biorthogonal and nonuniform lapped transforms for transform coding with reduced blocking and ringing artifacts," IEEE Trans. Signal Processing, vol. 46, pp. 1043-1053, Apr. 1998.

[2] — , "Enhancing the performance of subband audio coders for speech signals," in Proc. Int. Symp. Circuits and Systems'98, June 1998, pp. 90-101.

[3] R. L. de Queiroz, "Uniform filter banks with nonuniform bands: Postprocessing design," in Proc. ICASSP, vol. a, Apr. 1998, pp. 1341-1344.

[4] —-, "Approximating lapped transforms through unitary postprocessing," IEEE Signal Processing Lett., vol. 8, pp. 307-309, Dec. 2001.

[5] J.-J. Lee and B. G. Lee, "A design of nonuniform cosine-modulated filter banks," IEEE Trans. Circuits Syst. II, vol. 42, pp. 732-737, Nov. 1995. 
[6] J. Li, T. Q. Nguyen, and S. Tantaratana, "A simple design method for near-perfect reconstruction nonuniform filter banks," IEEE Trans. Signal Processing, vol. 45, pp. 2105-2109, Aug. 1997.

[7] Z. Xiong and H. S. Malvar, "A nonuniform modulated complex lapped transform," IEEE Signal Processing Lett., vol. 8, pp. 257-260, Sept. 2001.

[8] R. A. Gopinath and C. S. Burrus, "Some results in the theory of modulated filter banks and modulated wavelet tight frames," Appl. Comput. Harmon. Anal., vol. 2, pp. 303-326, Mar. 1995.
[9] P. P. Vaidyanathan, Multirate Systems and Filter Banks. Englewood Cliffs, NJ: Prentice-Hall, 1993.

[10] O. A. Niamut. (2001, Aug.) The design of arbitrary nonuniform cosine-modulated filter banks. Delft Univ. Technol., Delft, The Netherlands. [Online]. Available: http://www-ict.its.tudelft.nl/ omar/documents/SPL00148/ncmfb_report.zip. 